\title{
Black elderberry extract attenuates inflammation and metabolic dysfunction in diet-induced obese mice
}

\author{
Nicholas J. Farrell, Gregory H. Norris, Julia Ryan, Caitlin M. Porter, Christina Jiang and \\ Christopher N. Blesso* \\ Department of Nutritional Sciences, University of Connecticut, Storrs, CT 06269, USA
}

(Submitted 15 May 2015 - Final revision received 2 July 2015 - Accepted 7 July 2015 - First published online 28 August 2015)

\begin{abstract}
Dietary anthocyanins have been shown to reduce inflammation in animal models and may ameliorate obesity-related complications. Black elderberry is one of the richest sources of anthocyanins. We investigated the metabolic effects of anthocyanin-rich black elderberry extract (BEE) in a diet-induced obese C57BL/6J mouse model. Mice were fed either a low-fat diet ( $n$ 8), high-fat lard-based diet (HFD; $n$ 16), HFD $+0.25 \%(\mathrm{w} / \mathrm{w})$ BEE $(0 \cdot 25 \%$-BEE; $n$ 16) or HFD $+1.25 \%$ BEE $(1 \cdot 25 \%$-BEE; $n$ 16) for 16 weeks. The $0 \cdot 25 \%$ BEE $(0 \cdot 034 \%$ anthocyanin, $\mathrm{w} / \mathrm{w})$ and $1.25 \%$ BEE $(0.17 \%$ anthocyanin, w/w) diets corresponded to estimated anthocyanin doses of 20-40 mg and 100-200 mg per kg of body weight, respectively. After 16 weeks, both BEE groups had significantly lower liver weights, serum TAG, homoeostasis model assessment and serum monocyte chemoattractant protein-1 compared with HFD. The $0 \cdot 25 \%$-BEE also had lower serum insulin and TNF $\alpha$ compared with HFD. Hepatic fatty acid synthase mRNA was lower in both BEE groups, whereas PPAR $\gamma 2$ mRNA and liver cholesterol were lower in $1.25 \%$ BEE, suggesting decreased hepatic lipid synthesis. Higher adipose PPAR $\gamma$ mRNA, transforming growth factor $\beta$ mRNA and adipose tissue histology suggested a pro-fibrogenic phenotype that was less inflammatory in $1.25 \%$-BEE. Skeletal muscle mRNA expression of the myokine IL-6 was higher in 0.25\%-BEE relative to HFD. These results suggest that BEE may have improved some metabolic disturbances present in this mouse model of obesity by lowering serum TAG, inflammatory markers and insulin resistance.
\end{abstract}

Key words: Black elderberry extract: Obesity: Insulin resistance: Diet-induced obese mice: Inflammation: Molecular nutrition

According to the WHO, estimated worldwide rates of overweight and obesity are 39 and $13 \%$, respectively ${ }^{(1)}$. Obese individuals have shortened life expectancies ${ }^{(2)}$; however, they do not typically die of obesity itself but rather obesity-related comorbidities, such as CVD, diabetes and certain types of cancers ${ }^{(3,4)}$. The adipose dysfunction and excessive ectopic lipid accumulation in tissues in obesity promotes an inflammatory state that is thought to be an underlying cause of these obesity-related comorbidities ${ }^{(5,6)}$. Therefore, methods that target and lower inflammation may be effective at preventing obesity-related comorbidities. Anthocyanins, a class of polyphenol belonging to the flavonoid family, are dietary bioactives whose intake has been shown to be inversely associated with inflammation and insulin resistance in humans $^{(7)}$. Cyanidin 3-glucoside (C3G), a major anthocyanin in nature, has been shown to enhance adipocyte function and protect adipocytes from metabolic stress in vitro, by enhancing PPAR $\gamma$ activity and inhibition of forkhead box $\mathrm{O} 1^{(8-10)}$. In animal models, dietary C3G fed at $0 \cdot 2 \%$ of the diet $(w / w)$ for 5 weeks has been shown to improve insulin sensitivity and adipose tissue inflammation in diet-induced obese C57BL/6 mice $^{(11)}$ and genetically diabetic mice ${ }^{(11,12)}$. Purified anthocyanins have also displayed protective effects against hepatic steatosis ${ }^{(11)}$ and non-alcoholic steatohepatitis ${ }^{(13)}$ in mouse models. In human clinical studies, purified anthocyanin supplementation (300-320 mg/d) has been shown to improve systemic markers of inflammation in healthy ${ }^{(14)}$ and hypercholesterolaemic adults ${ }^{(15)}$ compared with placebo. However, humans do not consume purified anthocyanins in isolation, and there is evidence of synergistic effects of different anthocyanins when used in combination in vitro ${ }^{(15)}$. Therefore, it is crucial to investigate obesity-protective effects of anthocyanins from whole foods, extracts and as isolated compounds.

Black elderberry (Sambucus nigra) contains one of the highest anthocyanin contents reported in foods $(1316 \mathrm{mg} / 100 \mathrm{~g}$ fresh

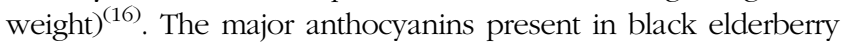
include C3G and cyanidin 3-sambubioside ${ }^{(17,18)}$. Black elderberry is commonly consumed in European cultures in wines and other processed beverages ${ }^{(19)}$, with similar products available in the USA. The berry has been used for centuries in traditional

Abbreviations: BEE, black elderberry extract; C3G, cyanidin 3-glucoside; CLS, crown-like structures; HFD, high-fat diet; HOMA-IR, homoeostasis model assessment of insulin resistance; LFD, low-fat diet; LPL, lipoprotein lipase; MCP-1, monocyte chemoattractant protein-1; TGF $\beta$, transforming growth factor $\beta$.

* Corresponding author: C. N. Blesso, fax +1 860486 3674, email christopher.blesso@uconn.edu 
medicine throughout European and native American cultures ${ }^{(19)}$; however, research on its therapeutic potential is limited. Therefore, we investigated the effects of anthocyanin-rich black elderberry extract (BEE) on the metabolic disturbances associated with obesity using the high-fat diet (HFD)-induced obese C57BL/6J mouse model. We hypothesised that BEE feeding would attenuate the low-grade inflammation and insulin resistance in this mouse model of obesity.

\section{Methods}

\section{Animals and diets}

HFD-fed C57BL/6 mice were used as a diet-induced model of obesity. Male C57BL/6J mice ( 8 weeks of age, $n 56$ ) were obtained from The Jackson Laboratory and allowed to acclimate to the animal facility for 2 weeks before being fed one of four experimental diets for 16 weeks: low-fat diet control group (LFD; $10 \%$ energy as fat; $n$ 8) (Harlan Teklad; TD.08806); HFD control group (60\% energy as fat; $n$ 16) (Harlan Teklad; TD.06414); HFD with $0.25 \%$ of BEE added by weight (0.25\%-BEE; $60 \%$ energy as fat; $n$ 16); and HFD with $1.25 \%$ of BEE added by weight (1.25\%-BEE; $60 \%$ energy as fat; $n$ 16). Spray-dried BEE ( $S$. nigra) (standardised to $13 \%$ anthocyanins) on maltodextrin as an excipient was kindly provided by Artemis International. A single batch of BEE was obtained from the supplier. BEE anthocyanins were previously characterised by HPLC and shown to be primarily in the form of cyanidin 3-sambubioside and $\mathrm{C}_{3} \mathrm{G}^{(18)}$. The $0.25 \%$-BEE and $1.25 \%$-BEE diets corresponded to $0.034 \%$ anthocyanin $(\mathrm{w} / \mathrm{w})$ and $0.17 \%$ anthocyanin $(\mathrm{w} / \mathrm{w})$ in diets, respectively. The estimated anthocyanin doses were $20-40 \mathrm{mg}$ (0.25\%-BEE) and 100-200 mg (1.25\%-BEE) per kg of body weight after accounting for weight gain throughout the study. Carbohydrate content of diets containing BEE was adjusted to match control HFD composition by replacing maltodextrin with BEE. The $0.25 \%$-BEE diet corresponds to a human dose of approximately $150 \mathrm{mg}$ of anthocyanin/d, which can easily be achieved with regular consumption of fruits such as berries ${ }^{(20)}$. The $1.25 \%$-BEE diet corresponds to about $1 / 2$ serving ( $60 \mathrm{~g}$ ) of black elderberry fruit ${ }^{(16)}$ (approximately $740 \mathrm{mg}$ of anthocyanin/d) for a $70 \mathrm{~kg}$ person. Food intake and body weight were assessed weekly. Fresh diet was provided to mice twice per week. After 16 weeks on experimental diets, mice were fasted for $6-8 \mathrm{~h}$ before blood collection by cardiac puncture and euthanasia. Blood was allowed to clot at room temperature for $30 \mathrm{~min}$ before serum was isolated by centrifugation (10000 $\boldsymbol{g}$ for $10 \mathrm{~min}$ at $4^{\circ} \mathrm{C}$ ) and then stored at $-80^{\circ} \mathrm{C}$. Tissues were perfused with saline before being harvested, snap-frozen in liquid $\mathrm{N}_{2}$ and stored at $-80^{\circ} \mathrm{C}$. Liver and adipose tissues were fixed in $10 \%$ neutral-buffered formalin for at least $48 \mathrm{~h}$ before histological analysis. All mice were housed in a temperature-controlled room and maintained in a $12 \mathrm{~h}$ light $-12 \mathrm{~h}$ dark cycle at the University of Connecticut-Storrs vivarium. The Animal Care and Use Committee of the University of Connecticut-Storrs approved all procedures used in the current study.

\section{Serum biochemical analysis}

Total cholesterol, NEFA, TAG, glucose and alanine aminotransferase (ALT) were measured using enzymatic assays, as described $^{(18)}$. Fasting insulin, monocyte chemoattractant protein-1 (MCP-1), IL-6, TNF $\alpha$, adiponectin, resistin and plasminogen activator inhibitor (PAI) were measured by Luminex/ xMAP magnetic bead-based multiplexing assays using MAGPIX instrumentation (EMD Millipore). The homoeostasis model assessment of insulin resistance (HOMA-IR) equation was used to estimate insulin resistance based on fasting serum insulin and glucose measurements ${ }^{(21)}$.

\section{Tissue lipid extraction and analysis}

Hepatic lipids were extracted using methods previously reported $^{(18)}$. Briefly, the lipids were extracted with chloroform: methanol (2:1), dried under $\mathrm{N}_{2}$ at $60^{\circ} \mathrm{C}$ and solubilised in Triton $\mathrm{X}-100$ before being analysed for cholesterol and TAG by enzymatic methods.

\section{RNA isolation, CDNA synthesis and real-time quantitative $R T-P C R$}

Total RNA was isolated from liver, skeletal muscle and adipose tissues using TRIzol reagent (Life Technologies). RNA was then DNase I-treated and reverse transcribed using the iScript cDNA synthesis kit (Bio-Rad). Gene expression was measured by real-time quantitative RT-PCR using SYBR Green (Bio-Rad) and a CFX96 real-time PCR detection system (Bio-Rad). A detailed list of all primer sequences used in quantitative RT-PCR analysis is provided in the online Supplementary material (online Supplementary Table S1). Liver mRNA expression data were normalised to the geometric mean of the internal controls glyceraldehyde 3-phosphate dehydrogenase and ribosomal protein large P0. The geometric mean of glyceraldehyde 3-phosphate dehydrogenase ribosomal protein large P0 and $\beta$-actin was used as an internal control for adipose and skeletal muscle mRNA analysis. Expression relative to the internal controls was determined using the $2^{-\Delta \Delta C_{t}}$ method.

\section{Histological analysis of tissues}

Formalin-fixed liver and epididymal adipose tissue were embedded in paraffin and cut into $5 \mu \mathrm{m}$ sections before staining. Liver sections were stained with haematoxylin-eosin (H\&E), whereas adipose sections were H\&E-stained or subjected to Masson's trichrome staining to visualise connective tissue. All histological procedures were conducted at the Connecticut Veterinary Medical Diagnostic Laboratory. The stained tissue sections were viewed under bright-field microscopy at $200 \times$ magnification, and images were taken with AxioCam ICc3 (Zeiss). The extent of macrophage infiltration into adipose tissue was assessed by the manual counting of crown-like structures (CLS) (three slides per animal) performed by a technician blinded to group assignment.

\section{Statistical analysis}

One-way ANOVA was used to detect differences between groups with post hoc multiple comparisons (Tukey's test) when appropriate $(P<0.05$ deemed significant). GraphPad Prism 
version 6 software was used to conduct all statistical analysis. Data are reported as mean values and standard error of means.

\section{Results}

\section{Effects of black elderberry extract on food intake and body weight}

Although there were no differences in food intake (Fig. 1(A)) between any of the four groups, the HFD groups had greater body weight and weight change after 16 weeks than the LFD group (Fig. 1(B) and (C)). There were no differences in body weight or weight change among the HFD groups. Liver weights were approximately $13 \%$ lower in both the $0.25 \%$ - and $1.25 \%$-BEE groups relative to the HFD control group (Fig. 1(D)).

\section{Black elderberry extract lowers serum TAG, inflammatory markers and insulin resistance}

Serum markers after 16 weeks are presented in Table 1. Serum TAG was significantly reduced compared with HFD control in the $0.25 \%$-BEE and $1.25 \%$-BEE groups by 25 and $30 \%$, respectively. There was a significant increase in total serum cholesterol and ALT in the HFD groups relative to LFD control; however, there were no differences within the HFD groups. No differences in serum NEFA were observed between the groups. The HFD control group displayed significant elevations in MCP-1, IL-6, TNF $\alpha$, resistin and PAI-1, as well as a reduction in adiponectin compared with LFD control (Fig. 2(A) and (B)). BEE feeding attenuated the HFD-dependent increase in several serum inflammatory cytokines/chemokines. There were

\section{(A)}

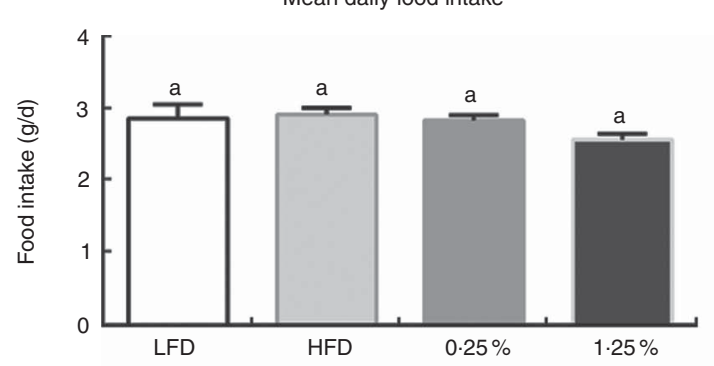

(C)

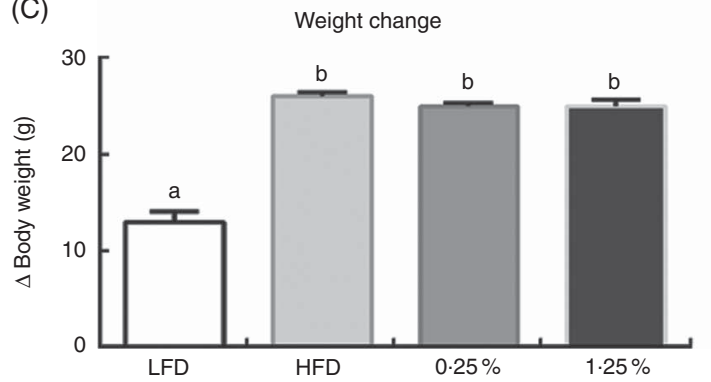

significant reductions in MCP-1 (-37\%) and TNF $\alpha$ (-47\%) with the $0.25 \%$-BEE dose and a significant reduction in MCP-1 $(-30 \%)$ with the $1.25 \%$-BEE dose (Fig. 2(A)). However, BEE feeding did not attenuate the effects of HFD on serum IL-6, resistin, PAI-1 and adiponectin (Fig. 2(A) and (B)). Fasting serum insulin was $32 \%$ lower in the $0.25 \%$-BEE group compared with HFD control (Fig. 2(C)), and HOMA-IR, a metric of insulin resistance, was significantly lower in both BEE groups compared with HFD control (Fig. 2(D)).

\section{Black elderberry extract reduces hepatic cholesterol and lipogenic gene expression}

HFD feeding significantly increased hepatic lipids compared with LFD control, suggesting the development of hepatic steatosis (Fig. 3(B) and (C)). This observation was confirmed by examination of H\&E-stained livers, in which extensive lipid droplet accumulation was seen in the HFD groups (Fig. 3(A)). Hepatic lipid accumulation with HFD appeared to be attenuated by BEE feeding (Fig. 3(C)). Hepatic cholesterol in the $1.25 \%$-BEE group was significantly lower (-32\%) than HFD control (Fig. 3(B)). Hepatic TAG did not differ significantly between HFD groups, although BEE groups tended to be lower (Fig. 3(C)). After hepatic lipid analysis, we performed real-time quantitative RT-PCR analysis to assess hepatic mRNA expression of lipid metabolism-related genes. Expression of the lipogenic gene fatty acid synthase (Fas) was significantly reduced in both BEE-fed groups, whereas PPAR 2 was significantly reduced only in the $1.25 \%$-BEE group relative to the HFD control (Fig. 4), corresponding with histological and biochemical analysis.

(B)
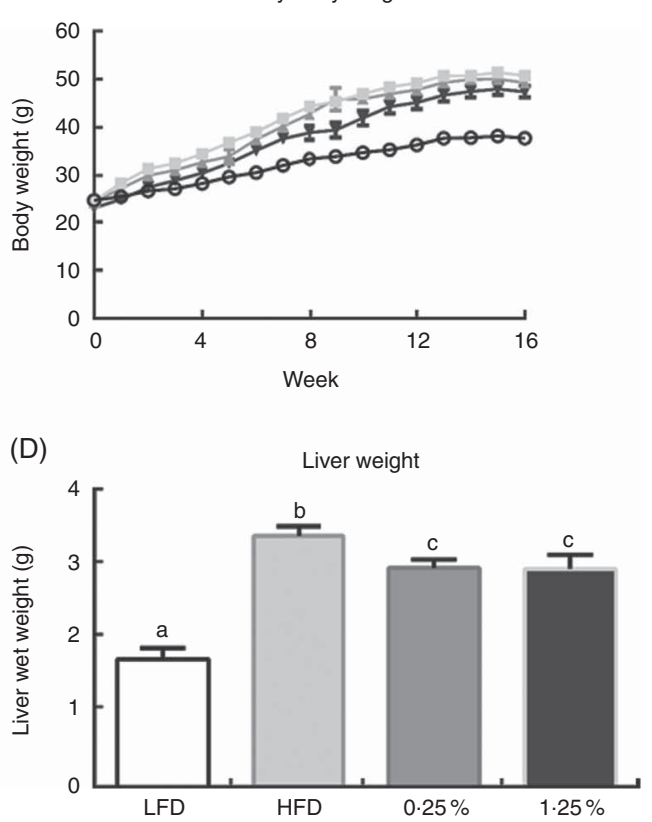

Fig. 1. Black elderberry extract (BEE) reduces liver weight with no change in food intake or weight gain. Food intake (A) and body weight of animals (B) were measured weekly. Mean weight change was calculated after 16 weeks (C), and liver weight was measured at the time of killing (D) ( $n$ 8-16/group). Values are means with their standard errors represented by vertical bars. ${ }^{\text {a,b,c }}$ Mean values with unlike letters were significantly different using post hoc comparisons $(P<0.05)$. HFD, high-fat diet; LFD, low-fat diet. $\_$, LFD; —-, HFD; —, $0.25 \%$ BEE; $\neg-1.25 \%$ BEE. 
Table 1. Serum markers of C57BL/6J mice after 16 weeks (Mean values with their standard errors)

\begin{tabular}{|c|c|c|c|c|c|c|c|c|c|c|c|}
\hline & \multirow[b]{2}{*}{$n$} & \multicolumn{2}{|c|}{ Total cholesterol $(\mathrm{mmol} / \mathrm{l})$} & \multicolumn{2}{|c|}{ HDL-cholesterol ( $\mathrm{mmol} / \mathrm{l})$} & \multicolumn{2}{|c|}{ TAG $(\mathrm{mmol} / \mathrm{l})$} & \multicolumn{2}{|c|}{$\mathrm{NEFA}(\mathrm{mmol} / \mathrm{l})$} & \multicolumn{2}{|c|}{ ALT (U/l) } \\
\hline & & Mean & SEM & Mean & SEM & Mean & SEM & Mean & SEM & Mean & SEM \\
\hline LFD & 8 & $3.43^{\mathrm{a}}$ & 0.11 & $2 \cdot 26^{a}$ & 0.13 & $0.37^{a}$ & 0.06 & 0.62 & 0.05 & $15 \cdot 93^{\mathrm{a}}$ & $2 \cdot 71$ \\
\hline HFD & 16 & $4.88^{\mathrm{b}}$ & 0.26 & $3.42^{\mathrm{b}}$ & 0.20 & $0.55^{\mathrm{b}}$ & 0.03 & 0.66 & 0.03 & $43 \cdot 21^{b}$ & 3.13 \\
\hline $0.25 \%-B E E$ & 16 & $4.65^{\mathrm{b}}$ & 0.24 & $3 \cdot 11^{\mathrm{b}}$ & 0.18 & $0.41^{\mathrm{a}}$ & 0.03 & 0.66 & 0.03 & $39.36^{b}$ & 3.16 \\
\hline $1.25 \%-B E E$ & 16 & $4 \cdot 69^{\mathrm{b}}$ & 0.18 & $3 \cdot 34^{\mathrm{b}}$ & 0.20 & $0.38^{\mathrm{a}}$ & 0.05 & 0.60 & 0.04 & $41 \cdot 26^{b}$ & 4.08 \\
\hline
\end{tabular}

ALT, alanine aminotransferase; BEE, black elderberry extract; HFD, high-fat diet; LFD, low-fat diet.

${ }^{a, b}$ Mean values within a column with unlike superscript letters were significantly different using post hoc comparisons $(P<0.05)$.

(A)

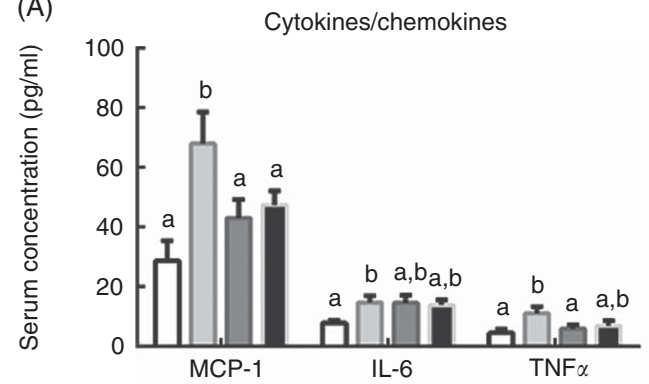

(C)

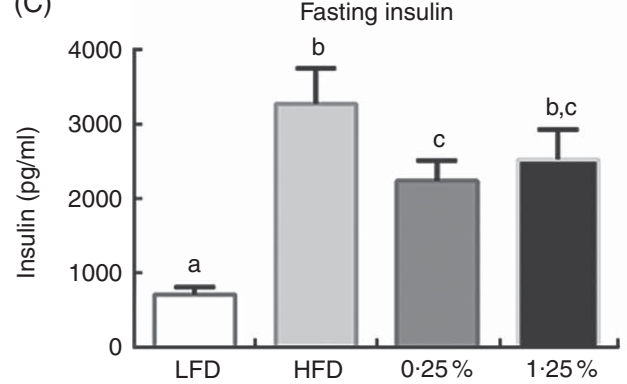

(B)

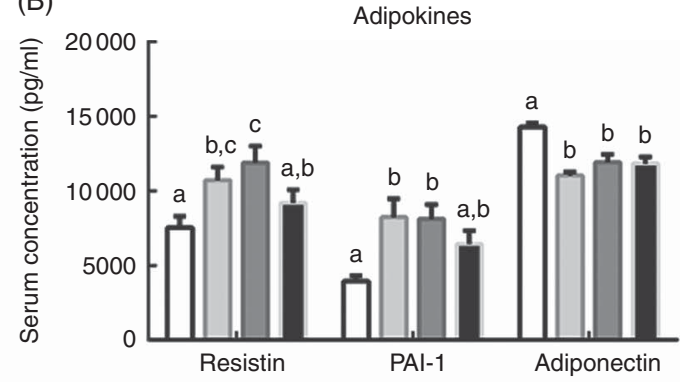

(D)

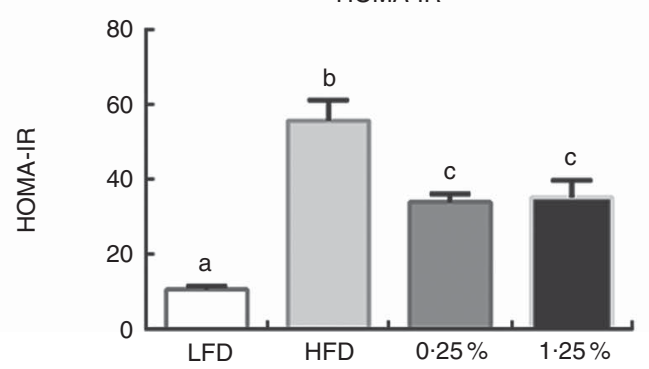

Fig. 2. Black elderberry extract (BEE) reduces serum inflammation and insulin resistance. Serum cytokines/chemokines, adipokines and insulin were examined by multiplexing assays $(\mathrm{A}-\mathrm{C})$. homoeostasis model assessment of insulin resistance $(\mathrm{HOMA}-\mathrm{IR})$ was calculated as $((\mathrm{glucose} \mathrm{mmol} / \mathrm{l}) \times($ insulin $\mathrm{mU} / \mathrm{I}) / 22.5)(\mathrm{D})(n \mathrm{8}-16 /$ group). HFD, high-fat diet; LFD, low-fat diet; MCP-1, monocyte chemoattractant-1; PAI-1, plasminogen activator inhibitor-1. Values are means with their standard errors represented by vertical bars. ${ }^{\text {a,b,c }}$ Mean values with unlike letters were significantly different using post hoc comparisons (P<0.05). $\square$, LFD; $\square$, HFD; $\square, 0.25 \%$ BEE;

口, $1.25 \%$ BEE.

\section{Black elderberry extract does not attenuate adipose tissue macrophage infiltration and fibrosis}

Compared with the LFD group, all HFD groups had a noticeable increase in macrophage infiltration indicated by H\&E staining, with an increased number of CLS (Fig. 5(A) and (B)). There were no differences in CLS among the HFD groups, as all groups appeared to have extensive immune cell infiltration and large pockets without adipocytes, suggesting fibrosis. The adipose tissue became markedly fibrotic in the HFD groups, as shown by Masson's trichrome blue staining of connective tissue (Fig. 5(C)). Indeed, the large pockets that lacked adipocytes stained strongly for connective tissue, and this staining appeared to be greatest in the $1.25 \%$-BEE group relative to the other HFD groups. To further examine adipose tissue, real-time quantitative RT-PCR was performed to examine changes in mRNA expression between HFD groups (Fig. 5(D)). In the 1.25\%-BEE group, there were significant increases in expression of PPAR $\gamma$ and a PPAR $\gamma$ target gene, adipocyte protein 2, compared with the HFD control group.
Another PPAR $\gamma$ target gene, lipoprotein lipase, was significantly higher in the $1.25 \%$-BEE group relative to the $0.25 \%$-BEE group. F4/80, a macrophage marker, was also more highly expressed in the $1.25 \%$-BEE group compared with the HFD control and 0.25\%-BEE groups. Interestingly, although the $1.25 \%$ BEE group had greater F4/80 mRNA expression, TNF $\alpha$ expression was significantly reduced compared with the 0.25\%-BEE group, suggesting that the macrophages were less inflammatory. The expression of CD11c, a phenotypic marker of 'classically activated' M1-like inflammatory macrophages, was not different between any of the groups, suggesting that $1.25 \%$-BEE macrophages are of the M2-like 'alternatively activated' phenotype that remodel adipose tissue and are not inflammatory. Supporting this notion, mRNA expression of transforming growth factor $\beta$ (TGF $\beta$ ), a pro-fibrogenic cytokine, was increased in the $1.25 \%$-BEE group relative to the other HFD groups. In addition, collagen VI $\alpha 3$ (Col6a3), a downstream target of TGF $\beta$ that is highly enriched in adipose tissue, was significantly increased in the $1.25 \%$-BEE group relative to the $0.25 \%$-BEE group. 
(A)

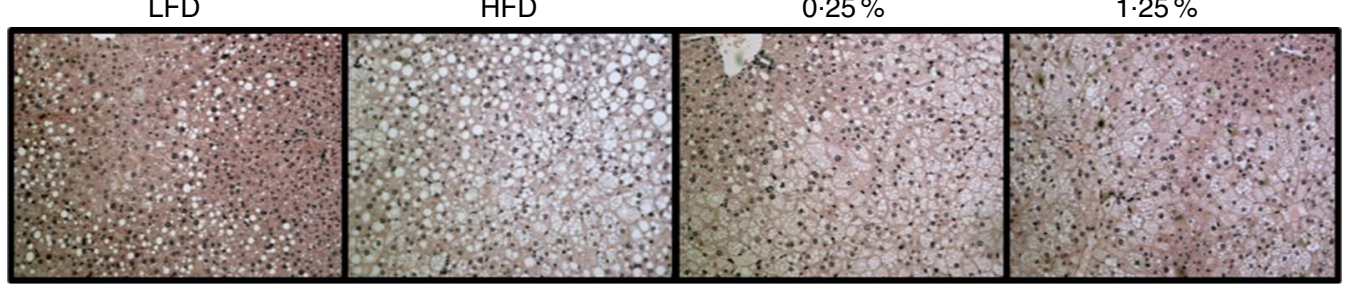

(B)

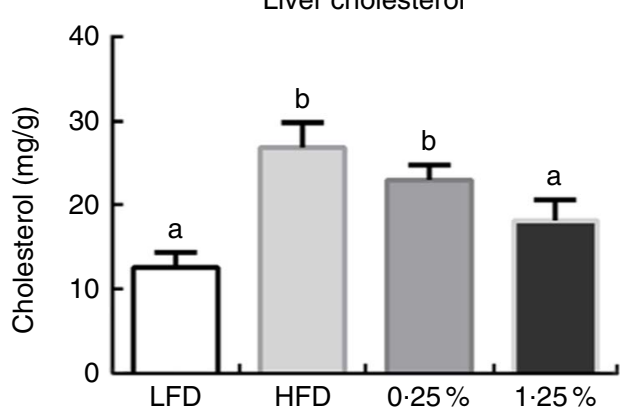

(C)

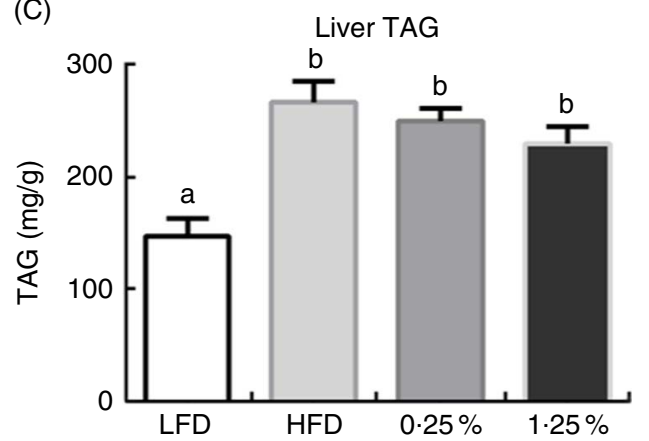

Fig. 3. Effect of black elderberry extract (BEE) on hepatic lipids and steatosis development. Liver haematoxylin-eosin (H\&E) histology was performed as described in the Methods section (A) ( $n$ 8/group). Hepatic lipids were extracted with chloroform-methanol $(2: 1)$, dried under nitrogen at $60^{\circ} \mathrm{C}$ and solubilised in Triton $\mathrm{X}-100$, as described in the Methods section. Cholesterol and TAG were measured by enzymatic methods (B, C) ( $n$ 8-16/group). Values are means with their standard errors represented by vertical bars. ${ }^{a, b}$ Mean values with unlike letters were significantly different using post hoc comparisons $(P<0.05)$. HFD, high-fat diet; LFD, low-fat diet.

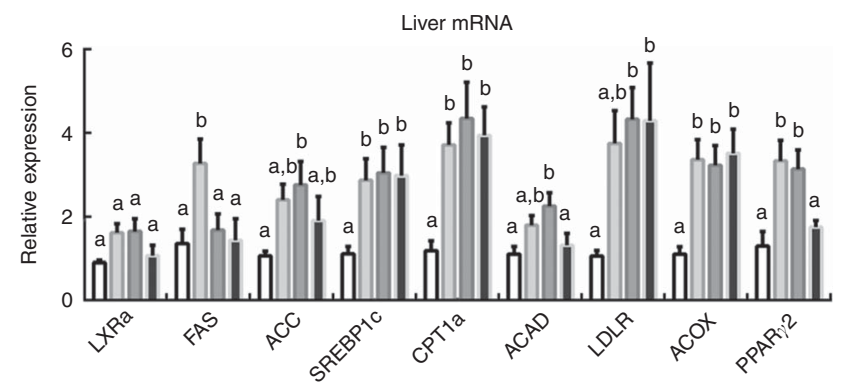

Fig. 4. Black elderberry extract (BEE) reduces lipogenic mRNA expression in the liver. Hepatic mRNA expression was measured by real-time quantitative RTPCR. Data were normalised to endogenous reference gene expression (n 8-16/group). ACAD, acyl-CoA dehydrogenase; ACC, acetyl-CoA carboxylase; ACOX, acyl-CoA oxidase 1; CPT1a, carnitine palmitoyltransferase 1a; FAS, fatty acid synthase; HFD, high-fat diet; LDLR, LDL receptor; LFD, low-fat diet; LXRa, liver $\mathrm{X}$ receptor $a$; SREBP1c, sterol regulatory element-binding protein 1c. Values are means with their standard errors represented by vertical bars. ${ }^{a, b}$ Mean values with unlike letters were significantly different using post hoc comparisons $(P<0.05)$. $\square$, LFD; $\square$, HFD; $\square, 0.25 \% ; \square, 1.25 \%$

\section{Black elderberry extract alters lipid metabolism-related gene expression but does not attenuate skeletal muscle inflammation}

To examine skeletal muscle inflammation and metabolic function, mRNA expression in quadriceps was measured (Fig. 6). Compared with LFD control, HFD feeding resulted in higher mRNA expression of skeletal muscle MCP-1 and the macrophage marker, CD68, suggesting that the obese state caused inflammation in this tissue. BEE feeding was unable to alter MCP-1 or CD68 expression compared with HFD control. There was also a marked increase in the expression of lipid metabolism-related genes acyl-CoA dehydrogenase, $L p l$ and acyl-CoA oxidase (Acox) with high-fat feeding compared with LFD. $L p l$ and Acox expression were decreased significantly in the $1.25 \%$-BEE group relative to the HFD control. IL-6, a myokine and regulator of substrate utilisation, was increased by $2 \cdot 7$-fold in the $0.25 \%$-BEE group compared with the HFD control group.

\section{Discussion}

Targeting the excessive lipid accumulation and inflammation in obesity may lead to successful therapies that reduce the prevalence of obesity-related comorbidities ${ }^{(5)}$. Anthocyanins are dietary bioactives that have been shown to reduce inflammation and insulin resistance in obese animals ${ }^{(11,12)}$. Black elderberry is a berry rich in anthocyanins, but there is limited research examining its effects on inflammation in chronic disease models. In this study, C57BL/6J mice fed BEE were shown to have an attenuation of insulin resistance and systemic inflammation compared with HFD controls. BEE-fed mice were also shown to have lower serum TAG and modest reductions in hepatic lipids compared with HFD controls. These changes suggest that BEE may have potential in ameliorating the lipotoxicity and inflammation present in obesity.

Anthocyanins have been shown to have limited bioavailability and are not found in the serum in significant quantities $(<1 \%)$ upon feeding ${ }^{(22)}$. Because of this, some have questioned how the consumption of anthocyanin-rich foods may be eliciting their effects ${ }^{(23)}$. Recently, Czank et al. ${ }^{(24)}$ showed that ingestion of $500 \mathrm{mg}$ of ${ }^{13} \mathrm{C}$-isotopically labelled C3G by humans was about $12 \%$ bioavailable (excreted via urine and breath) in 
(A)

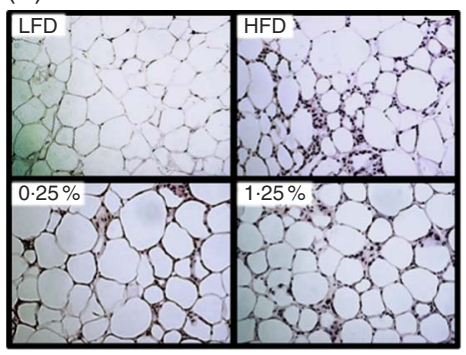

(C)

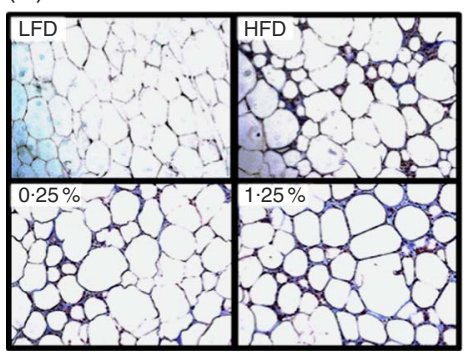

(B)

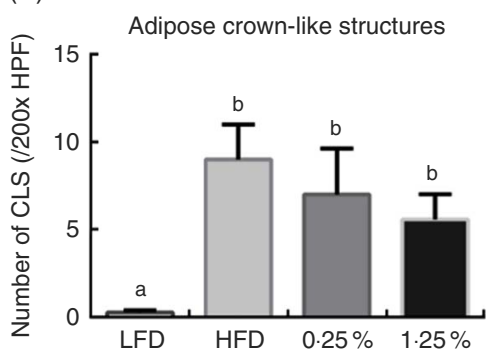

(D)

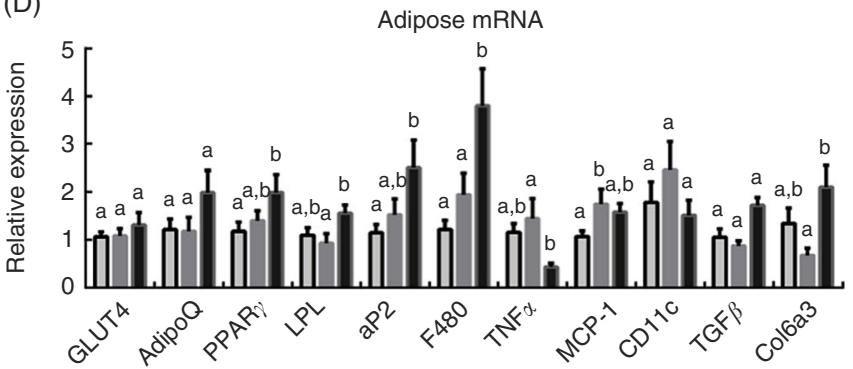

Fig. 5. Effect of black elderberry extract (BEE) on adipose tissue inflammation and fibrosis. Epididymal adipose haematoxylin-eosin (H\&E) (A) and Masson's trichrome $(C)$ staining was performed as described in the Methods section. Crown-like structures (CLS) were manually counted from adipose H\&E stains and averaged across three random 200x high-powered fields (HPF) (B) ( $n$ 8/group). Adipose mRNA expression was measured by real-time quantitative RT-PCR. Data were normalised to endogenous reference gene expression (D) ( $n$ 16/group). AdipoQ, adiponectin; aP2, adipocyte protein 2; CD11c, integrin, aX (complement component 3 receptor 4 subunit); Col6a3, collagen, type VI, a3; F480, EGF-like module-containing mucin-like hormone receptor-like 1; HFD, high-fat diet; LFD, low-fat diet; LPL, lipoprotein lipase; MCP-1, monocyte chemoattractant-1; TGF $\beta$, transforming growth factor- $\beta$. Values are means with their standard errors represented by vertical bars. ${ }^{\mathrm{a}, \mathrm{b}}$ Mean values with unlike letters were significantly different using post hoc comparisons $(P<0.05)$. $\square, \mathrm{HFD} ; \square, 0.25 \% \mathrm{BEE}$; $\square, 1.25 \%$ BEE.

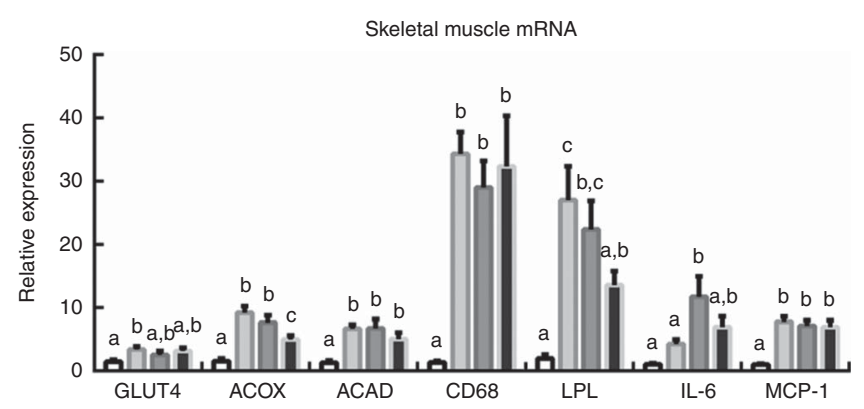

Fig. 6. Effect of black elderberry extract (BEE) on skeletal muscle gene expression. Skeletal muscle mRNA was measured by real-time quantitative RT-PCR. Data were normalised to endogenous reference gene expression ( $n$ 8-16/group). ACAD, acyl-CoA dehydrogenase; ACOX, acyl-CoA oxidase 1; CD68, cluster of differentiation 68; HFD, high-fat diet; LFD, low-fat diet; LPL, lipoprotein lipase; MCP-1, monocyte chemoattractant-1. Values are means with their standard errors represented by vertical bars. ${ }^{\mathrm{a}, \mathrm{b}, \mathrm{c}}$ Mean values with unlike letters were significantly different using post hoc comparisons (P<0.05). $\square$, LFD; $\square, \mathrm{HFD}$; $\square, 0.25 \%$ BEE; 口, $1.25 \%$ BEE.

the form of metabolites. C3G is degraded to protocatechuic acid (PCA), either spontaneously or after catabolism by the gut microbiota, which can then be absorbed and enter the bloodstream $^{(25)}$. Compared with intact C3G, much higher concentrations of these phenolic degradation products and their phase II conjugates were found in serum, urine and faeces in human subjects after ${ }^{13} \mathrm{C}$-labelled C3G ingestion ${ }^{(24)}$. In this case, it is likely that the metabolites of anthocyanins are primarily responsible for their physiological effects.

In the current study, both groups of BEE-fed mice were found to have improvements in many serum markers of obesityrelated metabolic complications. BEE-fed mice had 25-30\% reductions in fasting serum TAG compared with HFD control, although no other significant differences were observed in serum lipids. BEE appeared to attenuate systemic inflammation, as mice fed the lower dosage of BEE had $>30 \%$ lower serum MCP-1 and TNF $\alpha$, whereas only MCP-1 reached significance in the higher dosage group. Both BEE-fed groups displayed 20 and $40 \%$ reductions in fasting insulin and HOMA-IR, respectively, which suggests that BEE reduced insulin resistance in these obese mice. However, future studies should examine insulin resistance using glucose tolerance tests or clamps, as these would provide greater insight into the metabolic effects of BEE in obese conditions.

BEE-fed mice also displayed modest changes in markers of hepatic steatosis that increase with diet-induced obesity. BEE-fed mice were found to have $13 \%$ lower liver weights compared with HFD control. We observed dose-related effects of BEE on hepatic lipids and lipogenic gene expression. There appeared to be a modest attenuation of hepatic steatosis in the $1.25 \%$-BEE group 
compared with the HFD control, with noticeably smaller lipid droplets, suggesting less macrosteatosis. A significant reduction of hepatic cholesterol by $32 \%$ and a non-significant $16 \%$ attenuation in hepatic TAG in the $1.25 \%$-BEE group confirm this observation. These changes may be explained in part by reductions in hepatic FAS and PPAR $\gamma 2$ mRNA in the $1.25 \%$-BEE group. No changes in $\beta$-oxidation-related gene expression were observed among the HFD groups, suggesting that BEE influenced TAG synthesis rather than oxidation.

The effects of BEE feeding on adipose tissue did not appear to explain the differences in serum markers between HFD groups. Despite no changes in the epididymal adipose CLS count among the HFD groups, the $1.25 \%$-BEE displayed a $211 \%$ increase in the mRNA expression of the pan-macrophage marker F4/80. Although F4/80 expression was increased, the 1.25\%-BEE group adipose tissue did not appear inflamed, having significantly lower TNF $\alpha$ expression than the $0.25 \%$-BEE group, and this approached significance compared with HFD control. Furthermore, CD11c, a marker of pro-inflammatory 'classically activated' M1-like macrophages, was not increased in the $1.25 \%$-BEE group. Adipose mRNA expression of PPAR $\gamma$ and TGF $\beta$, which are induced in M2-like 'alternatively activated' macrophages ${ }^{(26,27)}$, was significantly increased in the $1.25 \%$-BEE groups compared with HFD control. Potentially, the macrophages in the $1.25 \%$-BEE adipose tissue were more of an M2-like phenotype, where they would be pro-resolving and anti-inflammatory. Expression levels of PPAR $\gamma$ target genes $(a P 2, L P L)$ were increased in $1.25 \%$-BEE adipose relative to other HFD groups, suggesting that PPAR $\gamma$ activity was increased. PPAR $\gamma$ activation in macrophages has been shown to induce an anti-inflammatory M2-like phenotype that improves metabolic function in obese mice ${ }^{(26,28)}$. Alternatively activated M2-like macrophages have also been shown to stimulate fibrogenesis in vitro via production of $\mathrm{TGF} \beta^{(29)}$. Connective tissue staining of sectioned adipose tissue appeared to be somewhat greater in the $1.25 \%$-BEE group, which also displayed a higher expression of Col6a3, a fibrogenic gene that was shown to be induced downstream of $\mathrm{TGF} \beta$ signalling in vitro ${ }^{(30)}$. In human obese subjects, adipose tissue macrophages that were present in fibrotic areas and not in CLS were shown to be primarily M2-like macrophages, which produce TGF $\beta$ and increase adipocyte collagen $\mathrm{VI}^{(27)}$. Thus, despite the strong induction of $\mathrm{F} 4 / 80 \mathrm{mRNA}$ in the $1.25 \%$-BEE adipose, the infiltrated macrophages do not appear to be inflammatory and may be of the M2-like anti-inflammatory and pro-fibrogenic phenotype.

In skeletal muscle, we observed dose-related effects of BEE on the expression of several fatty acid metabolism genes. Skeletal muscle mRNA expression levels of LPL and ACOX were reduced by 50 and $46 \%$ in the $1.25 \%$-BEE group compared with HFD control, which indicates major changes in fatty acid metabolism. However, these genes were highly induced in all HFD groups compared with LFD, suggesting that these decreases with the $1.25 \%$-BEE dose were indicative of an attenuation of the HFD effect. This may possibly be explained by reduced fatty acid availability to the skeletal muscle as a consequence of greater adipose PPAR $\gamma$ activation and adipose fatty acid buffering capacity. With long-term HFD feeding in mice ( $>12$ weeks), skeletal muscle can exhibit extensive macrophage infiltration and inflammation similar to adipose tissue $^{(31)}$. BEE feeding was unable to attenuate the apparent macrophage infiltration (>30-fold increases in CD68 mRNA expression) and inflammation with HFD feeding ( $>$ five-fold increases in MCP-1 mRNA expression). Of interest was the $2 \cdot 7$-fold increase in IL-6 mRNA expression in the $0.25 \%$-BEE group compared with HFD control. IL-6 is a myokine and somewhat controversial in regard to its effects in skeletal muscle, systemic glucose tolerance, and insulin resistance ${ }^{(32,33)}$. Although traditionally viewed as an inflammatory cytokine that increases hepatic insulin resistance, increasing evidence points to a beneficial metabolic role in promoting glucose uptake and fatty acid oxidation in skeletal muscle ${ }^{(34,35)}$. This may be significant in regulating systemic glucose tolerance, as the skeletal muscle is the major organ involved in whole-body glucose disposal in humans ${ }^{(36)}$.

Our findings are consistent with other reports that have fed anthocyanins and anthocyanin-rich foods to obese rodent models. Guo et al. $^{(11)}$ observed significant reductions in plasma insulin, HOMA-IR, MCP-1 and TNF $\alpha$, with no changes in IL-6 after feeding diet-induced obese C57BL/6 mice with $0.2 \%(\mathrm{w} / \mathrm{w})$ C3G for 5 weeks. We reported reductions in fasting insulin and HOMA-IR by $20-30$ and $40 \%$, respectively. DeFuria et al. ${ }^{(37)}$ also observed decreases in insulin resistance in C57BL/6J mice fed blueberry powder in the diet at $4 \%$ by weight (about $0 \cdot 12 \%$ anthocyanins) for 8 weeks. Similarly, Chuang et $a l .{ }^{(38)}$ reported an improvement in glucose tolerance in diet-induced obese C57BL/6 mice fed 3\% (w/w) grape powder for 18 weeks. The significant reductions in serum MCP-1 (37 and $30 \%$ in $0.25 \%$-BEE and $1.25 \%$-BEE groups, respectively, relative to HFD control) and TNF $\alpha$ ( $47 \%$ in the $0.25 \%$-BEE group relative to HFD control) are comparable to the reductions of $30-50 \%$ reported by Chuang et $a l .{ }^{(38)}$ in serum inflammatory markers. Therefore, the results that we report in this study are consistent with previous findings on the efficacy of anthocyanin-rich foods in ameliorating inflammation and insulin resistance in obese rodent models.

In conclusion, BEE-fed mice had reduced serum inflammatory markers and insulin resistance, as measured by HOMA-IR. BEE-fed mice had lower fasting TAG and modest reductions in hepatic lipids, possibly explained by reductions in hepatic FAS and PPAR 2 . Despite a lack of difference in CLS in the HFD groups, the $1.25 \%$-BEE-fed mice appeared to have more macrophage infiltration in the adipose tissue, as demonstrated by greater F4/80 mRNA expression. These macrophages do not appear to be inflammatory, however, and may be depositing more collagen. Across the tissues, changes in mRNA expression in the BEE-fed groups suggest differences in fatty acid metabolism including potentially decreased lipogenesis in the liver and increased adipogenesis in the adipose tissue. Both BEE doses appear to attenuate some of the complications induced by HFD feeding, although the $1.25 \%(\mathrm{w} / \mathrm{w})$ dose does not appear to improve upon the serum changes observed with the $0 \cdot 25 \%(\mathrm{w} / \mathrm{w})$ dose and may even cause complications in the adipose because of fibrogenic effects. Overall, BEE appeared to attenuate systemic inflammation and insulin resistance that occurs with diet-induced obesity in this mouse model, but further research is warranted on black elderberry consumption and effects in humans. 


\section{Acknowledgements}

This work was supported by USDA AFRI (CONS2014-06619) and USDA Hatch (CONS00934) grants to C. N. B.

N. F. conducted the research, analysed the data and wrote the paper; G. N. conducted the research and provided input for the paper; J. R., C. M. P. and C. J. conducted the research; C. N. B. designed the research, analysed the data and had primary responsibility for final content. All authors read and approved the final manuscript.

There are no conflicts of interest.

\section{Supplementary material}

For supplementary material/s referred to in this article, please visit http://dx.doi.org/doi:10.1017/S0007114515002962

\section{References}

1. World Health Organization (2015) Obesity and overweight, fact sheet no. 311. http://www.who.int/mediacentre/factsheets/ fs311/en (accessed May 2015).

2. Peeters A, Barendregt JJ, Willekens F, et al. (2003) Obesity in adulthood and its consequences for life expectancy: a lifetable analysis. Ann Intern Med 138, 24-32.

3. Must A, Spadano J, Coakley EH, et al. (1999) The disease burden associated with overweight and obesity. JAMA 282, 1523-1529.

4. Haslam DW \& James WP (2005) Obesity. Lancet 366, 1197-1209.

5. Hotamisligil GS (2006) Inflammation and metabolic disorders. Nature 444, 860-867.

6. Guilherme A, Virbasius JV, Puri V, et al. (2008) Adipocyte dysfunctions linking obesity to insulin resistance and type 2 diabetes. Nat Rev Mol Cell Biol 9, 367-377.

7. Jennings A, Welch AA, Spector T, et al. (2014) Intakes of anthocyanins and flavones are associated with biomarkers of insulin resistance and inflammation in women. J Nutr 144, 202-208.

8. Guo H, Ling W, Wang Q, et al. (2008) Cyanidin 3-glucoside protects 3T3-L1 adipocytes against $\mathrm{H} 2 \mathrm{O} 2-$ or TNF-alphainduced insulin resistance by inhibiting c-Jun NH2-terminal kinase activation. Biochem Pharmacol 75, 1393-1401.

9. Guo H, Guo J, Jiang X, et al. (2012) Cyanidin-3-O-betaglucoside, a typical anthocyanin, exhibits antilipolytic effects in 3T3-L1 adipocytes during hyperglycemia: involvement of FoxO1-mediated transcription of adipose triglyceride lipase. Food Chem Toxicol 50, 3040-3047.

10. Scazzocchio B, Vari R, Filesi C, et al. (2011) Cyanidin-3-Obeta-glucoside and protocatechuic acid exert insulin-like effects by upregulating PPARgamma activity in human omental adipocytes. Diabetes 60, 2234-2244.

11. Guo H, Xia M, Zou T, et al. (2012) Cyanidin 3-glucoside attenuates obesity-associated insulin resistance and hepatic steatosis in high-fat diet-fed and $\mathrm{db} / \mathrm{db}$ mice via the transcription factor FoxO1. J Nutr Biochem 23, 349-360.

12. Sasaki R, Nishimura N, Hoshino H, et al. (2007) Cyanidin 3glucoside ameliorates hyperglycemia and insulin sensitivity due to down-regulation of retinol binding protein 4 expression in diabetic mice. Biochem Pharmacol 74, 1619-1627.

13. Tang X, Shen T, Jiang X, et al. (2015) Purified anthocyanins from bilberry and black currant attenuate hepatic mitochondrial dysfunction and steatohepatitis in mice with methionine and choline deficiency. J Agric Food Chem 63 , $552-561$.
14. Karlsen A, Retterstol L, Laake P, et al. (2007) Anthocyanins inhibit nuclear factor-kappaB activation in monocytes and reduce plasma concentrations of pro-inflammatory mediators in healthy adults. J Nutr 137, 1951-1954.

15. Zhu Y, Ling W, Guo H, et al. (2013) Anti-inflammatory effect of purified dietary anthocyanin in adults with hypercholesterolemia: a randomized controlled trial. Nutr Metab Cardiovasc Dis 23, 843-849.

16. Perez-Jimenez J, Neveu V, Vos F, et al. (2010) Systematic analysis of the content of 502 polyphenols in 452 foods and beverages: an application of the phenol-explorer database. J Agric Food Chem 58, 4959-4969.

17. Wu X, Gu L, Prior RL, et al. (2004) Characterization of anthocyanins and proanthocyanidins in some cultivars of Ribes, Aronia, and Sambucus and their antioxidant capacity. J Agric Food Chem 52, 7846-7856.

18. Farrell N, Norris G, Lee SG, et al. (2015) Anthocyanin-rich black elderberry extract improves markers of HDL function and reduces aortic cholesterol in hyperlipidemic mice. Food Funct 6, 1278-1287.

19. Charlebois D, Byers P, Finn C, et al. (2010) Elderberry: botany, horticulture, potential. Hortic Rev (Am Soc Hortic Sci) 37, 213-280.

20. Wu X, Beecher GR, Holden JM, et al. (2006) Concentrations of anthocyanins in common foods in the United States and estimation of normal consumption. J Agric Food Chem $\mathbf{5 4}$, 4069-4075.

21. Matthews DR, Hosker JP, Rudenski AS, et al. (1985) Homeostasis model assessment: insulin resistance and beta-cell function from fasting plasma glucose and insulin concentrations in man. Diabetologia 28, 412-419.

22. Manach C, Williamson G, Morand C, et al. (2005) Bioavailability and bioefficacy of polyphenols in humans. I. Review of 97 bioavailability studies. Am J Clin Nutr 81, 230S-242SS

23. Lotito SB \& Frei B (2006) Consumption of flavonoid-rich foods and increased plasma antioxidant capacity in humans: cause, consequence, or epiphenomenon? Free Radic Biol Med 41, $1727-1746$.

24. Czank C, Cassidy A, Zhang Q, et al. (2013) Human metabolism and elimination of the anthocyanin, cyanidin-3-glucoside: a (13)C-tracer study. Am J Clin Nutr 97, 995-1003.

25. Vitaglione P, Donnarumma G, Napolitano A, et al. (2007) Protocatechuic acid is the major human metabolite of cyanidin-glucosides. J Nutr 137, 2043-2048.

26. Bouhlel MA, Derudas B, Rigamonti E, et al. (2007) PPARgamma activation primes human monocytes into alternative M2 macrophages with anti-inflammatory properties. Cell Metab 6, 137-143.

27. Spencer M, Yao-Borengasser A, Unal R, et al. (2010) Adipose tissue macrophages in insulin-resistant subjects are associated with collagen VI and fibrosis and demonstrate alternative activation. Am J Physiol Endocrinol Metab 299, E1016-E1027.

28. Odegaard JI, Ricardo-Gonzalez RR, Goforth MH, et al. (2007) Macrophage-specific PPARgamma controls alternative activation and improves insulin resistance. Nature $\mathbf{4 4 7}$, $1116-1120$.

29. Song E, Ouyang N, Horbelt M, et al. (2000) Influence of alternatively and classically activated macrophages on fibrogenic activities of human fibroblasts. Cell Immunol 204, $19-28$.

30. Verrecchia F, Chu ML \& Mauviel A (2001) Identification of novel TGF-beta /Smad gene targets in dermal fibroblasts using a combined cDNA microarray/promoter transactivation approach. J Biol Chem 276, 17058-17062. 
31. Patsouris D, Cao JJ, Vial G, et al. (2014) Insulin resistance is associated with MCP1-mediated macrophage accumulation in skeletal muscle in mice and humans. PLOS ONE 9, e110653.

32. Munoz-Canoves P, Scheele C, Pedersen BK, et al. (2013) Interleukin-6 myokine signaling in skeletal muscle: a doubleedged sword? FEBS J 280, 4131-4148.

33. Wallenius V, Wallenius K, Ahren B, et al. (2002) Interleukin6-deficient mice develop mature-onset obesity. Nat Med 8, 75-79.

34. Carey AL, Steinberg GR, Macaulay SL, et al. (2006) Interleukin-6 increases insulin-stimulated glucose disposal in humans and glucose uptake and fatty acid oxidation in vitro via AMP-activated protein kinase. Diabetes $\mathbf{5 5}$ 2688-2697.
35. Glund S, Deshmukh A, Long YC, et al. (2007) Interleukin-6 directly increases glucose metabolism in resting human skeletal muscle. Diabetes 56, 1630-1637.

36. Shulman GI, Rothman DL, Jue T, et al. (1990) Quantitation of muscle glycogen synthesis in normal subjects and subjects with non-insulin-dependent diabetes by ${ }^{13} \mathrm{C}$ nuclear magnetic resonance spectroscopy. $N$ Engl J Med 322, 223-228.

37. DeFuria J, Bennett G, Strissel KJ, et al. (2009) Dietary blueberry attenuates whole-body insulin resistance in high fat-fed mice by reducing adipocyte death and its inflammatory sequelae. J Nutr 139, 1510-1516.

38. Chuang CC, Shen W, Chen H, et al. (2012) Differential effects of grape powder and its extract on glucose tolerance and chronic inflammation in high-fat-fed obese mice. J Agric Food Chem 60, 12458-12468. 